VOL. 44 (1991) [203-205]

\title{
ON RINGS GENERATING ATOMS OF LATTICES OF SPECIAL AND SUPERNILPOTENT RADICALS
}

\section{HaLina France-JaCKSON}

\begin{abstract}
This note is to indicate a nonsemiprime ring $R$ such that the smallest supernilpotent (respectively special) radical containing the ring $R$ is an atom of the lattice of all supernilpotent (respectively special) radicals. This gives a positive answer to Puczylowski's and Roszkowska's question.
\end{abstract}

It is known [1] that the collections of all supernilpotent (that is hereditary and containing the prime radical $\beta$ ) and all special radicals of associative rings form complete lattices. We denote these lattices by $K$ and $S p$ respectively. The smallest supernilpotent (respectively special) radical containing a ring $A$ will be denoted by $\overline{1} A$ (respectively $\widehat{I} A$ ).

It is easy to check that if $s$ is a supernilpotent radical then the class of all prime and $s$-semisimple rings is a special class and the upper radical $\hat{s}$ determined by this class is the smallest special radical containing $s$.

A prime ring $R \neq 0$ is called a *-ring $[2,3,4]$ if for every non-zero ideal $I$ of $R$, the factor ring $R / I$ is $\beta$-radical.

The problem of a description of atoms in $K$ and $S p$ was raised in [1]. Then it was studied in $[\mathbf{2}, \mathbf{3}, \mathbf{4}, \mathbf{5}]$. Among others, the following was proved:

PROPOSITION 1. [5, Proposition 12]. If $s$ is an atom of $K$ then $\hat{s}$ is an atom of $S p$.

Proposttion 2. [3, Theroem 1]. If $A$ is a ${ }^{*}$-ring then $\overline{1} A$ is an atom of $K$.

Proposition 3. [4, Theorem 1]. If $A$ is a *-ring then $\hat{1} A$ is an atom of $S p$.

Proposition 4. [3, Proposition 1]. If $A$ is a ${ }^{*}$-ring then a ring $R$ is an $\overline{1} A$ radical if and only if every non-zero semiprime homomorphic image of $R$ has a non-zero ideal isomorphic to an accessible subring of $A$.

In view of the abovementioned results, Puczylowski and Roszkowska have put a natural question [5, Question 6] whether there exists a non- *-ring $R$ such that $\overline{1} R$ is an atom of $K$ or $\widehat{1} R$ is an atom of $S p$. The aim of this note is to give a positive answer to this question. For this purpose we use the ring $R$ described in the following:

Received 11 September 1990

Copyright Clearance Centre, Inc. Serial-fee code: 0004-9729/91 \$A2.00+0.00. 
EXAMPLE: Let us consider the matrix ring

$$
R=\left\{\left(\begin{array}{ll}
r & a \\
0 & r
\end{array}\right) \mid r, a \in A\right\},
$$

where $A$ is a simple (that is, idempotent without nontrivial ideals) ring. Evidently, the subset

$$
H=\left\{\left(\begin{array}{ll}
0 & a \\
0 & 0
\end{array}\right) \mid a \in A\right\}
$$

is a non-zero zero-ring ideal of $R$ with $R / H \simeq A$. Thus $R$ is neither semiprime nor $\beta$-radical. In particular, $R$ is not a *-ring. Also the only ideals of $R$ are $\{0\}, H$ and $R$. Indeed, let $I \neq 0$ be an ideal of $R$ and $0 \neq\left(\begin{array}{ll}r & a \\ 0 & r\end{array}\right) \in I$. If $r=0$ then $a \neq 0$ and so $A a A$ is a non-zero ideal of $A$, since $A$ is semiprime. But $A$ is simple, so $A a A=A$. Thus for every $x \in A$ there exist $y, z \in A$ such that $x=\sum y a z$. Hence for every $x \in A$, we have

$$
\begin{aligned}
\left(\begin{array}{ll}
0 & x \\
0 & 0
\end{array}\right) & =\left(\begin{array}{cc}
0 & \sum y a z \\
0 & 0
\end{array}\right)=\sum\left(\begin{array}{cc}
0 & y a z \\
0 & 0
\end{array}\right) \\
& =\sum\left(\begin{array}{ll}
y & 0 \\
0 & y
\end{array}\right)\left(\begin{array}{ll}
0 & a \\
0 & 0
\end{array}\right)\left(\begin{array}{ll}
z & 0 \\
0 & z
\end{array}\right) \in I
\end{aligned}
$$

since $\left(\begin{array}{ll}0 & a \\ 0 & 0\end{array}\right) \in I$ and $I$ is an ideal of $R$. This implies $H \subseteq I$. Similarly, if $r \neq 0$ then there exists an element $b \in A$ such that $r b \neq 0$, since $A$ is semiprime. Consequently $A r b A$ is a non-zero ideal of a simple ring $A$ and thus $A r b A=A$. Hence, for every $x \in A$ there exist $y, z \in A$ such that $x=\sum y(r b) z$ and so we have

$$
\begin{aligned}
\left(\begin{array}{ll}
0 & x \\
0 & 0
\end{array}\right) & =\left(\begin{array}{cc}
0 & \sum y r b z \\
0 & 0
\end{array}\right)=\sum\left(\begin{array}{cc}
0 & y r b z \\
0 & 0
\end{array}\right) \\
& =\sum\left(\begin{array}{ll}
y & a \\
0 & y
\end{array}\right)\left(\begin{array}{ll}
r & a \\
0 & r
\end{array}\right)\left(\begin{array}{ll}
0 & b \\
0 & 0
\end{array}\right)\left(\begin{array}{ll}
z & 0 \\
0 & z
\end{array}\right) \in I,
\end{aligned}
$$

since $\left(\begin{array}{ll}r & a \\ 0 & r\end{array}\right) \in I$ and $I$ is an ideal of $R$. Thus, also in this case, $H \subseteq I$. This, however, implies $H=I$ or $I=R$, for otherwise $I / H$ would be a non-trivial ideal of $R / H$, which is impossible since $R / H \simeq A$ and $A$ is simple. Therefore $R / H \simeq A$ is the only non-zero semiprime homomorphic image of $R$ and, obviously, $A$ is an accessible subring of itself. Therefore, by Proposition $4, R$ is $\overline{1} A$-radical. Consequently $\overline{1} A \subseteq \overline{1} A$. On the other hand, $\beta \nsubseteq \overline{1} R$, since $R$ is a non-zero $\overline{1} R$-ring and $R$ is not a $\beta$-ring. This, and the fact that $\overline{1} A$ is an atom of $K$, by Proposition 2 , implies $\overline{1} R=\overline{1} A$. Hence $\overline{1} R$ is an atom of $K$. But then Proposition 1 gives immediately that $\hat{1} R$ is an atom of $S p$ which ends the proof. 


\section{REFERENCES}

[1] V.A. Andrunakievich and Yu. M. Rjabukhin, Radicals of Algebras and Structure Theory (Nauka, Moscow, 1979). (In Russian).

[2] H. France-Jackson, '"-rings and their radicals', Questiones Math. 8 (1985), 231-239.

[3] H. France-Jackson, 'On atoms of the lattice of supernilpotent radicals', Questiones Math. 10 (1987), 251-256.

[4] H. Korolczuk, 'A note on the lattice of special radicals', Bull. Acad. Polon. Sci. Ser. Sci. Math. 29 (1981), 103-104.

[5] E.R. Puczylowski and E. Roszkowska, 'Atoms of lattices of radicals of associative rings', Radical Theory, Proceedings of the 1988 Sendai Conference, 123-134.

Department of Mathematics

Vista University

Private Bag $\times 613$

Port Elizabeth 6000

South Africa 